\title{
Traumatismes de la femme enceinte
}

\section{Pregnant Trauma Patient}

\author{
C. Le Ray $\cdot$ M. Raux \\ (C) SFMU et Lavoisier SAS 2016
}

« On va recevoir une femme enceinte traumatisée ! ». Qui n'a jamais eu le ventre noué lorsqu'est tombée cette annonce, dont le contenu menace de transformer la garde aux urgences en véritable cauchemar?

Le traumatisé ? Le médecin urgentiste sait le prendre en charge. Il a appris à le faire, et il en voit souvent. Les grandes urgences de la femme enceinte ? Il a également appris à les prendre en charge à leur phase toute initiale, et il en voit au cours de son exercice. Le problème survient lors de la conjonction des deux phénomènes et que la femme enceinte devient la victime d'un traumatisme. «Femme enceinte traumatisée », c'est presque un oxymore. Parce que la population des traumatisés est très majoritairement masculine. Parce qu'une femme enceinte fait attention à elle et a tendance à se protéger des situations à risque de traumatisme, lorsqu'elle le peut. Parce que tout être doté d'humanité porte une attention particulière aux femmes enceintes. Du coup, c'est - heureusement - rare et le médecin urgentiste verra peu de femmes enceintes traumatisées au cours de son exercice médical. Battaloglu et al. rapportent une incidence de $1 \%$ de grossesses parmi les 15140 patientes prises en charge pour traumatisme au Royaume-Uni entre 2009 et 2014 [1].

L'angoisse que fait naître une telle annonce procède de multiples facteurs : la fragilité de l'être en développement, la peur des soignants de ne pas savoir faire, le contexte émotionnellement chargé, la rareté du tableau, laquelle compromet le développement de nos routines de prise en charge si rassurantes. La démarche diagnostique à mettre en œuvre chez une femme enceinte traumatisée est pourtant assez claire [2], quoique insuffisamment suivie par crainte des

C. Le Ray

Maternité Port-Royal, hôpital Cochin Broca Hôtel-Dieu, Assistance Publique-Hôpitaux de Paris, université Paris

Descartes ; DHU risques et grossesse, Paris, France

\section{Raux ( $\square)$}

Département d'anesthésie-réanimation, groupe hospitalier PitiéSalpêtrière Charles Foix, AP-HP, Sorbonne universités, UPMC Univ Paris 06, F-75013 Paris, France

e-mail : mathieu.raux@aphp.fr effets des rayons X sur le fœtus [3]. Après l'évaluation des conséquences du traumatisme sur la mère, vient le temps de l'évaluation des conséquences sur le foetus : sa vitalité, son rythme cardiaque, son placenta, le passage d'hématies fotales dans le sang maternel [4].

Quel est le devenir de la grossesse de ces patientes traumatisés ? Pour répondre à cette question, Richard-Jourjon et al. ont réalisé une étude rétrospective monocentrique sur quatre ans au sein de la maternité de niveau III d'un CHU disposant d'un centre de traumatologie [5]. Les auteurs ont inclus les femmes enceintes consultant aux urgences de la maternité dans les suites d'un traumatisme. Leur objectif était de décrire la population de ces patientes, leur prise en charge et leur devenir obstétrical ainsi que d'identifier les facteurs associés à une hospitalisation ou une complication obstétricale. Bien qu'il s'agisse d'une étude rétrospective, toutes les patientes ont bénéficié d'une même stratégie diagnostique obstétricale. Cette dernière associant un monitorage par cardiotocographie évaluant le rythme cardiaque fotal et les contractions utérines, une échographie fœtale évaluant la vitalité du foetus et un test de Kleihauer à la recherche d'hématies fotales dans la circulation maternelle.

Richard-Jourjon et al. retrouvent un faible collectif de femmes (144 sur 4 ans) [5]. Malheureusement, il n'a pas été possible de rapporter l'occurrence de la pathologie traumatique gestationnelle à l'ensemble de la population de patientes traumatisées prises en charge au CHU sur la même période. L'âge gestationnel médian était similaire à celui rapporté par d'autres auteurs [6] témoignant de traumatismes survenus au cours du troisième trimestre. Plus de la moitié de ces femmes enceintes présentaient au moins un facteur de risque de complication de leur grossesse. Les auteurs ont identifié des facteurs associés à l'hospitalisation parmi lesquels la localisation abdominale du traumatisme, l'âge gestationnel élevé, la présence de facteurs de risque de complication de la grossesse ou des anomalies du bilan réalisé à l'entrée. Ce dernier point est sans surprise, puisqu'il s'agit là d'un critère habituel d'hospitalisation.

Plus intéressant est le taux de complications obstétricales, évalué à $17 \%$ dont $12 \%$ d'accouchement prématurés. Cette 
fréquence relativement élevée d'accouchement prématuré justifie idéalement une prise en charge obstétricale adaptée à l'âge gestationnel, à savoir un transfert dans un centre ayant une maternité de type 3 (doté d'une réanimation néonatale) avant 32 semaines d'aménorrhée (SA) ou de type 2 (doté d'une néonatologie) avant 37 SA. Richard-Jourjon et al. rapportent plusieurs complications mettant potentiellement en jeu le pronostic vital de l'enfant : trois hématomes rétroplacentaires, deux ruptures utérines et deux morts fœtales dans leur série de 144 patientes [5]. Le pronostic fotal dépend évidemment de l'âge gestationnel, du type, du mécanisme et de la sévérité du traumatisme, mais celui-ci peut également être mis en jeu pour des traumatismes parfois considérés comme mineurs. Ainsi, devant tout traumatisme abdomino-pelvien chez une femme enceinte, l'hospitalisation devrait être la règle, en particulier lorsque la viabilité fotale est atteinte (24-25 SA). La prise en charge initiale pour le fœtus comprend au minimum un monitoring fœetal cardiotocographique, une échographie et un test de Kleihauer. Les morts fotales surviennent majoritairement en cas d'accidents de la voie publique, en particulier de haute cinétique [7]. Mais ce sont aussi ces accidents de la voie publique qui entraînent les lésions maternelles les plus graves. Dans les cas où le pronostic vital maternel est également mis en jeu, il semble évident que la prise en charge de la mère prime sur celle de l'enfant.

\section{Références}

1. Battaloglu E, McDonnell D, Chu J, et al (2016) Epidemiology and outcomes of pregnancy and obstetric complications in trauma in the United Kingdom. Injury 47:184-7

2. Mendez-Figueroa H, Dahlke, JD, Vrees RA, Rouse DJ (2013) Trauma in pregnancy: an updated systematic review. Am J Obstet Gynecol 209:1-10

3. Horstmann P, Larsen CF, Grønborg H (2014) Adherence to protocol in pregnant trauma patients? A 12-year retrospective study. Eur J Trauma Emerg Surg 40:561-6

4. Rozenberg A (2001) Polytraumatisme chez la femme enceinte. In: Beydon L, Carli P, Riou B. Traumatismes graves, Paris, Arnette 7:171-7

5. Vanessa Richard-Jourjon V, Dehours E, Parant M, et al (2016) Complications obstétricales des traumatismes de la femme enceinte : épidémiologie dans une maternité d'un CHU en France. Ann Fr Med Urg 6:307-12

6. Weiner E, Gluck O, Levy M, et al (2016) Obstetric and neonatal outcome following minor trauma in pregnancy. Is hospitalization warranted? Eur J Obstet Gynecol Reprod Biol 203:78-81

7. Weiss HB, Songer TJ, Fabio A (2001) Fetal deaths related to maternal injury. JAMA 286:1863-8 